\title{
Dynamics of $\mathrm{Li}$ ions in an intercalated polymer electrolyte: $A^{7}$ Li nuclear magnetic resonance study
}

\author{
N. Arun and S. Vasudevan ${ }^{\mathrm{a})}$ \\ Department of Inorganic and Physical Chemistry, Indian Institute of Science, Bangalore, India \\ K. V. Ramanathan ${ }^{\text {b) }}$ \\ Sophisticated Instruments Facility, Indian Institute of Science, Bangalore, India
}

\begin{abstract}
The ${ }^{7} \mathrm{Li}$ nuclear magnetic resonance spectra and spin-lattice relaxation rates of lithium ions in an intercalated polymer electrolyte, Li-polyethylene-oxide confined within the galleries of an inorganic layered solid $\mathrm{CdPS}_{3}$, are reported. The spectra show an unusual feature, the appearance above 330 $\mathrm{K}$ of quadrupolar satellites, whose splitting increases linearly with temperature, and their absence below this temperature. This behavior is also reflected in the temperature variation of the spin-lattice relaxation rates, which show a minimum at $300 \mathrm{~K}$ but above $330 \mathrm{~K}$ start decreasing again. Using an anisotropic quantum oscillator model, the observed variation in the ${ }^{7} \mathrm{Li}$ quadrupolar coupling constant is shown to arise from changes in the time average of the electric field gradient as seen by a ${ }^{7} \mathrm{Li}$ nucleus associated with mobile segments of the intercalated polymer.
\end{abstract}

\section{INTRODUCTION}

Ion-conducting, solvent-free polymer electrolytes based on alkali metal ions dissolved in polyethylene-oxide (PEO) and other polyethers have received considerable attention in the field of solid-state ionics. ${ }^{1-6}$ Nuclear magnetic resonance (NMR) studies have figured prominently in investigations of both the molecular structure and dynamics of the alkali ion in solid polymer electrolytes (SPEs), especially since ion mobilities are significant only in the amorphous phase above the glass transition temperature. It was early NMR studies on the onset of ${ }^{7} \mathrm{Li}$ and ${ }^{23} \mathrm{Na}$ NMR motional narrowing above the glass transition that highlighted the importance of polymer segmental motion to ion transport. ${ }^{7}$ The relative contribution of cations and anions to the measured conductivity, too, was established by NMR measurements. ${ }^{8}$ These measurements indicated that anions were mobile, in some cases more than the cation. Pulse field gradient measurements of diffusion of ions in the $\mathrm{LiCF}_{3} \mathrm{SO}_{3} / \mathrm{PEO} \mathrm{SPE}$, too, arrived at a similar conclusion. ${ }^{9}$

Dynamical information from the temperature variation of ${ }^{7} \mathrm{Li}$ and ${ }^{23} \mathrm{Na}$ linewidths have been reported. ${ }^{10-12}$ in SPEs. For $\mathrm{LiCF}_{3} \mathrm{SO}_{3}$ /polypropylene glycol (PPG) complexes the results indicate liquidlike behavior of the $\mathrm{Li}$ ions with the motion describable by a single-exponential correlation function. The spin-lattice relaxation mechanism was shown to be dominated by quadrupolar interactions between ${ }^{7} \mathrm{Li}$ nuclear moments and the electric field gradient (efg) due to the polymer lattice. Line shapes, however, were dominated by both dipolar and quadrupolar interactions. ${ }^{10}$ The motional parameters extracted from the spin-lattice relaxation times $T_{1}$ indicated that coupling between the mobile Li ions and the struc-

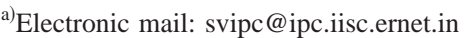

${ }^{b)}$ Electronic mail: kvr@sif.iisc.ernet.in
}

tural relaxation modes of the polymer chains plays an important role in the cation diffusion process. A detailed study of ${ }^{23} \mathrm{Na}$ NMR line shapes and spin-lattice relaxation times in $\mathrm{NaClO}_{4} / \mathrm{PPG}$ complexes has been reported. ${ }^{11,12}$ The temperature variation of $T_{1}$ showed more structure than expected for a typical viscous liquid, displaying a distinct change in behavior at a temperature $T_{c} \sim 1.2 T_{g}$, at which temperature a minimum in the linewidth is observed. The observation was interpreted as indicating a crossover between two distinct dynamical regimes: a regime above $T_{c}$ where there is a coupling of ionic motion to the polymer segmental motion and a regime below $T_{c}$ where decoupling occurs. The dynamics in the composite polymer electrolytes, PEO intercalated in montmorillonite and $\mathrm{Li}$-fluorohectorite, has been probed by ${ }^{7} \mathrm{Li}$ and ${ }^{2} \mathrm{H}$ NMR. ${ }^{13-15}$ Spin-lattice relaxation rate studies indicated that the fluctuating fields responsible for relaxation were the same for both the cation and polymer since the motional parameters, activation energies, and residence times were very similar. Ion hopping was shown to occur at rates much slower than polymer reorientation rates. ${ }^{14}$

In the preceding paper ${ }^{16}$ we presented spectroscopic evidence of the association of $\mathrm{Li}$ ions with mobile polymer segmental modes in the intercalated polymer electrolyte (IPE) $\mathrm{Cd}_{0.75} \mathrm{PS}_{3} \mathrm{Li}_{0.5}$ (PEO). ${ }^{17}$ The temperature variation of the ${ }^{7} \mathrm{Li} \mathrm{NMR}$ linewidths is observed to mirror the conductivity behavior; ${ }^{18}$ it exhibits a change in the mechanism of line narrowing from one with an Arrhenius temperature dependence at low temperatures to one with a Vogel-TammanFulcher (VTF) dependence at high temperatures. In addition, the ${ }^{7} \mathrm{Li}$ NMR spectra exhibit an unusual behavior-the appearance of quadrupolar satellites at high temperatures ( $T$ $>330 \mathrm{~K})$, where conductivity values are appreciable, and their absence at lower temperatures, where conductivity values are small. The high-temperature quadrupolar splitting 

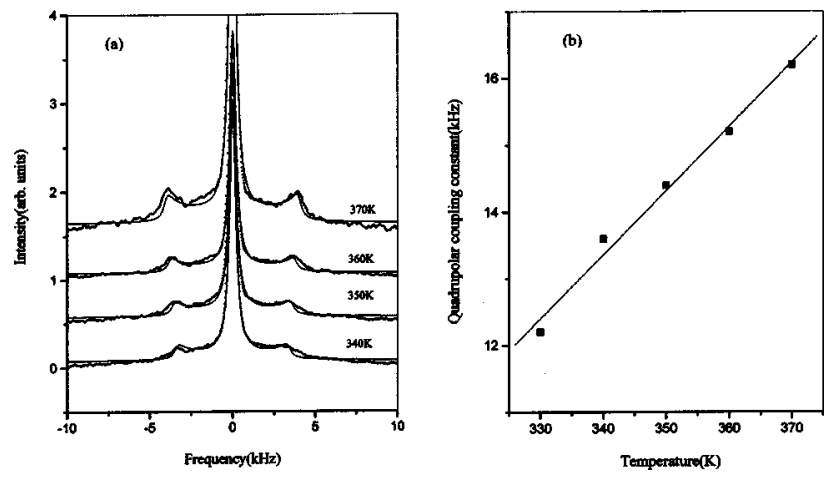

FIG. 1. (a) High-temperature ${ }^{7} \mathrm{Li}$ NMR spectra of $\mathrm{Cd}_{0.75} \mathrm{PS}_{3} \mathrm{Li}_{0.5}(\mathrm{PEO})$. The solid line is the fit to a theoretical quadrupolar powder pattern for a $I$ $=3 / 2$ nuclei. (b) Temperature variation of the ${ }^{7} \mathrm{Li}$ quadrupolar coupling constant.

shows a linear increase with temperature. The results were interpreted as signifying the solvation of $\mathrm{Li}$ ions by mobile segments of the intercalated PEO in a crown-ether-like environment at high temperatures. The corresponding $\mathrm{Li}-$ crown-ether complex (12-crown-4-lithium chloride) too shows a similar behavior-the appearance of a quadrupolar doublet at high temperatures with the splitting increasing with temperature. ${ }^{19}$

Here we show that a model in which the $\mathrm{Li}$ ion environment is such that it can vibrate anisotropically can satisfactorily explain the observed temperature dependence of the quadrupolar coupling constant. The magnitude of the electric field gradient depends on the anisotropy of the root mean square of the vibrational amplitude, which is temperature dependent. We also report the ${ }^{7} \mathrm{Li}$ spin-lattice relaxation rates as a function of temperature for the intercalated polymer electrolyte. The ${ }^{7} \mathrm{Li} T_{1}$ shows a minimum at $300 \mathrm{~K}$, but at higher temperatures, above $330 \mathrm{~K}$, exhibits an unusual behavior-a decrease in relaxation rates. This temperature is identical to the temperature at which the quadrupolar splitting appears in the ${ }^{7} \mathrm{Li}$ NMR spectra. We show that this behavior, too, follows as a consequence of the results derived from the anisotropic quantum Einstein oscillator model for quadrupolar coupling constants.

\section{RESULTS}

\section{A. ${ }^{7} \mathrm{Li}$ NMR}

The ${ }^{7} \mathrm{Li}$ NMR spectra of $\mathrm{Cd}_{0.75} \mathrm{PS}_{3} \mathrm{Li}_{0.5}(\mathrm{PEO})$ powder at different temperatures are reported in the preceding paper. ${ }^{16}$ The spectra did not exhibit satellites expected for an $I=3 / 2$ nucleus at temperatures below $330 \mathrm{~K}$. At temperatures above $330 \mathrm{~K}$, however, well-defined satellites were observed and the spectra in the temperature range 330-370 K resembled a quadrupolar powder pattern expected for a $I=3 / 2$ nucleus corresponding to an axial tensor. The high-temperature ( $T$ $>330 \mathrm{~K}$ ) spectra could be fitted satisfactorily to a theoretical powder pattern characteristic of a spin $I=3 / 2$ nucleus, ${ }^{20}$ convoluted with a Lorentzian. The experimental spectra (dotted line) along with the fits (solid line) at four different temperatures are shown in Fig. 1(a). The estimated quadrupolar cou-

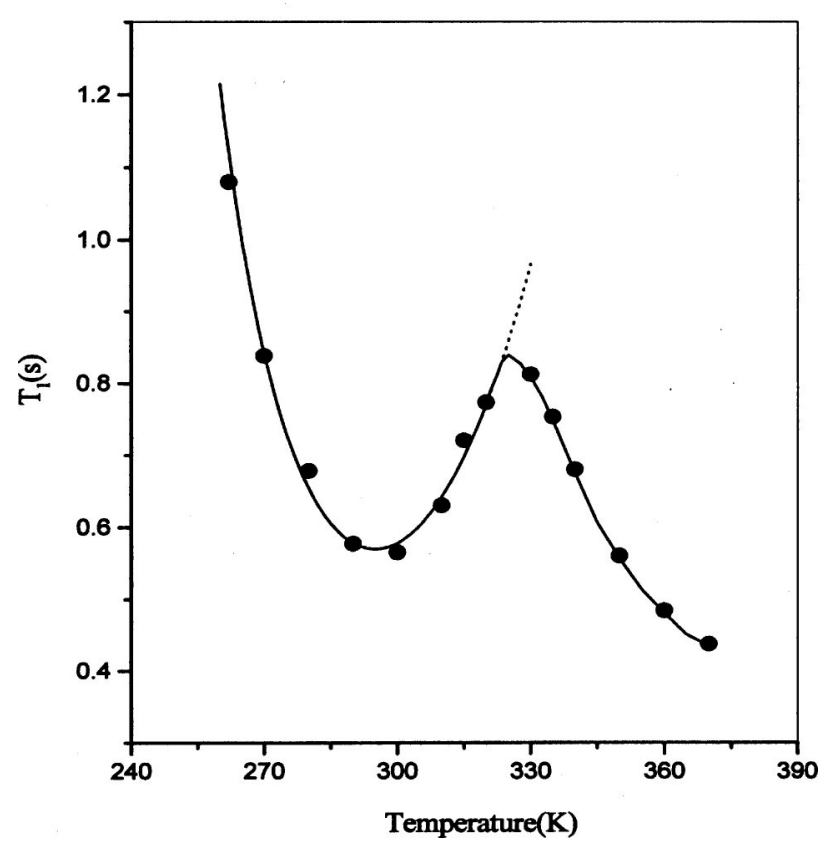

FIG. 2. ${ }^{7} \mathrm{Li}$ spin-lattice relaxation time of $\mathrm{Cd}_{0.75} \mathrm{PS}_{3} \mathrm{Li}_{0.5}(\mathrm{PEO})$ as a function of temperature. The solid line is a fit to the BPP expression [Eq. (18)]. The dashed line is the fit assuming the quadrupolar coupling constant $\omega_{Q}$ to be temperature independent.

pling constant $\left(e^{2} q Q / h\right)$ was found to have a linear dependence on temperature [Fig. 1(b)] ranging from $6.1 \mathrm{kHz}$ at $330 \mathrm{~K}$ to $8.1 \mathrm{kHz}$ at $370 \mathrm{~K}$.

\section{B. ${ }^{7} \mathrm{Li}$ spin-lattice relaxation}

The temperature variation of the ${ }^{7} \mathrm{Li}$ spin-lattice relaxation rates were measured over the range 250-370 K (Fig. 2 ). The ${ }^{7} \mathrm{Li}$ spin-lattice relaxation times at various temperatures were determined by sampling the fee-induction decay (FID) following the $\pi / 2$ pulse in the $\pi-\tau-\pi / 2$ "inversionrecovery" sequence followed by Fourier transform of the FIDs for 12 different values of $\tau$. At all temperatures, both above and below $330 \mathrm{~K}$, the recovery of the ${ }^{7} \mathrm{Li}$ magnetization to its equilibrium value was a single-exponential function of time, implying that all Li nuclei relax by a similar mechanism. This behavior is different from that in some SPEs where a double exponential is required to describe the magnetization recovery. The spin-lattice relaxation times show a classical parabolic minimum at $300 \mathrm{~K}$. The $T_{1}$ values, however, start decreasing above $330 \mathrm{~K}$. This temperature is identical to that at which the quadrupolar doublet appears in the ${ }^{7} \mathrm{Li}$ NMR spectra. The relaxation rates continue to decrease until the maximum temperature measured (370 K).

\section{DISCUSSION}

In the preceding paper ${ }^{16}$ the appearance of quadrupolar splitting above $330 \mathrm{~K}$ was interpreted as signifying the association of $\mathrm{Li}$ ions with mobile segments of the intercalated PEO in a crown-ether-like geometry. Here we show that the observed increase in the ${ }^{7} \mathrm{Li}$ quadrupole coupling constant with temperature (Fig. 1) may be explained as due to a 
change in the time average of the electric field gradients seen by an ${ }^{7} \mathrm{Li}$ nuclei vibrating anisotropically about its equilibrium value. ${ }^{21}$ In this section we derive an expression for the temperature dependence of the quadrupole coupling constants for an anisotropic quantum harmonic oscillator, where the force constants $k_{x}=k_{y} \neq k_{z}$, in the Einstein approximation.

Consider a nucleus that vibrates with simple harmonic motion in three dimensions about an equilibrium position $(0,0,0)$. If $V_{z z}(x, y, z)$ is the electric field gradient at the point $(x, y, z)$, then by Mclaurin's theorem it can be expressed in terms of $V_{z z}$, the field gradient at the origin, and derivatives of $V_{z z}$ (where $V_{z z}=\partial^{2} V / \partial z^{2}$ ). Thus $\left\langle V_{z z}\right\rangle$, the average value of $V_{z z}(x, y, z)$ sampled by the moving nucleus, is

$$
\begin{aligned}
\left\langle\frac{\partial^{2} V}{\partial z^{2}}\right\rangle= & \frac{\partial^{2} V}{\partial z^{2}}+\left\lfloor\frac{\partial^{3} V}{\partial z^{2} \partial x}\langle x\rangle+\frac{\partial^{3} V}{\partial z^{2} \partial y}\langle y\rangle+\frac{\partial^{3} V}{\partial z^{2} \partial z}\langle z\rangle \mid\right. \\
& +\frac{1}{2 !}\left\lfloor\frac{\partial^{4} V}{\partial z^{2} \partial x^{2}}\left\langle x^{2}\right\rangle+\frac{\partial^{4} V}{\partial z^{2} \partial y^{2}}\left\langle y^{2}\right\rangle\right. \\
& \left.+\frac{\partial^{4} V}{\partial z^{2} \partial z^{2}}\left\langle z^{2}\right\rangle\right\rfloor+\frac{1}{2 !}\left\lfloor\frac{\partial^{4} V}{\partial z^{2} \partial x \partial y}\langle x y\rangle+\cdots\right\rfloor \\
& +\frac{1}{3 !}\left\lfloor\frac{\partial^{5} V}{\partial z^{2} \partial x^{2} \partial y}\left\langle x^{2} y\right\rangle+\cdots\right\rfloor
\end{aligned}
$$

or

$$
\begin{aligned}
\left\langle V_{z z}\right\rangle= & V_{z z}+\left[V_{z z x}\langle x\rangle+V_{z z y}\langle y\rangle+V_{z z z}\langle z\rangle\right] \\
& +\frac{1}{2 !}\left[V_{z z x x}\left\langle x^{2}\right\rangle+V_{z z y y}\left\langle y^{2}\right\rangle+V_{z z z z}\left\langle z^{2}\right\rangle\right] \\
& +\frac{1}{2 !}\left[V_{z z x y}\langle x y\rangle+\cdots\right]+\frac{1}{3 !}\left[V_{z z x x y}\left\langle x^{2} y\right\rangle+\cdots\right],
\end{aligned}
$$

where $V_{z z x x}$, for example, represents $\partial^{4} V / \partial z^{2} \partial x^{2}$ and $\langle x\rangle$, $\langle y\rangle$, and $\langle z\rangle$ are the average values of the displacement of the nucleus in the $x, y$, and $z$ directions, respectively (notation from Ref. 21). The averages $\langle x\rangle,\langle y\rangle$, and $\langle z\rangle$ are equal to zero, but $\left\langle x^{2}\right\rangle,\left\langle y^{2}\right\rangle$, and $\left\langle z^{2}\right\rangle$ remain finite. In the case of axial symmetry, $\left\langle x^{2}\right\rangle=\left\langle y^{2}\right\rangle \neq\left\langle z^{2}\right\rangle$, Eq. (2) reduces to

$$
\left\langle V_{z z}\right\rangle=V_{z z}+\frac{1}{2}\left[\left(V_{z z x x}+V_{z z y y}\right)\left\langle x^{2}\right\rangle+V_{z z z z}\left\langle z^{2}\right\rangle\right] .
$$

From the Laplace equation we have

$$
-V_{z z z z}=V_{z z x x}+V_{z z y y} .
$$

Therefore Eq. (3) further simplifies as

$$
\left\langle V_{z z}\right\rangle=V_{z z}+\frac{1}{2} V_{z z z z}\left[\left\langle z^{2}\right\rangle-\left\langle x^{2}\right\rangle\right] .
$$

We evaluate the average root-mean-square (rms) displacements $\left\langle z^{2}\right\rangle$ and $\left\langle x^{2}\right\rangle$ for classical and quantum oscillators.

For a classical oscillator, considering each nuclei to be oscillating independently, the averages $\left\langle z^{2}\right\rangle$ and $\left\langle x^{2}\right\rangle$ can be calculated as the expectation value

$$
\left\langle x^{2}\right\rangle=\frac{\int_{-\infty}^{\infty} e^{-k_{x} x^{2} / 2 k_{B} T} x^{2} d x}{\int_{-\infty}^{\infty} e^{-k_{x} x^{2} / 2 k_{B} T} d x}=\frac{2 k_{B} T}{k_{x}},
$$

where $k_{B}$ is the Boltzmann constant and $k_{x}$ is the effective force constant in the $x$ direction. Similarly,

$$
\left\langle z^{2}\right\rangle=\frac{2 k_{B} T}{k_{z}} .
$$

On substituting the values of $\left\langle z^{2}\right\rangle$ and $\left\langle x^{2}\right\rangle$ in Eq. (4), $\left\langle V_{z z}\right\rangle$ becomes

$$
\left\langle V_{z z}\right\rangle=V_{z z}+\frac{V_{z z z z} k_{B}\left(k_{x}-k_{z}\right) T}{k_{x} k_{z}} .
$$

If the force constants are assumed to be independent of temperature, then it follows from Eq. (6) that

$$
\left\langle V_{z z}\right\rangle=V_{z z}+a T,
$$

where $a=V_{z z z z} k_{B}\left(k_{x}-k_{z}\right) / k_{x} k_{z}$. Since the quadrupole coupling constant is proportional to the average field gradient, Eq. (7) shows that this simple model predicts a linear temperature variation of the coupling constant with the slope proportional to the anisotropy of the force constants $\left(k_{x}\right.$ $-k_{z}$ ). This expression is similar to that derived in Ref. 21 .

For a quantum oscillator in a state with quantum number $n$ the mean value of the square of the displacement, $\left\langle x^{2}\right\rangle$, is given by $(n+1 / 2) \hbar / \sqrt{k_{x}}$, where $k_{x}$ is the force constant in the $x$ direction. ${ }^{22}$ The ensemble average $\left\langle x^{2}\right\rangle$ is given by

$$
\begin{aligned}
\left\langle x^{2}\right\rangle & =Z^{-1} \sum_{n=0}^{\infty}(n+1 / 2) \hbar / \sqrt{k_{x}} e^{-(n+1 / 2) \hbar \omega / k_{B} T} \\
& =Z^{-1} / \omega \sqrt{k_{x}} \sum_{n=0}^{\infty}(n+1 / 2) \hbar \omega e^{-(n+1 / 2) \hbar \omega / k_{B} T},
\end{aligned}
$$

where $Z$ is given by

$$
Z=\frac{e^{-\hbar \omega / 2 k_{B} T}}{1-e^{-\hbar \omega / k_{B} T}} .
$$

Equation (8) can be rewritten as

$$
\left\langle x^{2}\right\rangle=\frac{k_{B} T^{2}}{\omega \sqrt{k_{x}}} \frac{1}{Z} \frac{d Z}{d T} .
$$

Using Eq. (9) and after a simple calculation, one gets

$$
\left\langle x^{2}\right\rangle=\left[\frac{\hbar}{2 \sqrt{k_{x}}}+\frac{\hbar}{\sqrt{k_{x}} e^{\hbar \omega / k_{B} T-1}}\right]
$$

and similarly $\left\langle z^{2}\right\rangle$ is given by

$$
\left\langle z^{2}\right\rangle=\left[\frac{\hbar}{2 \sqrt{k_{z}}}+\frac{\hbar}{\sqrt{k_{z}} e^{\hbar \omega / k_{B} T}-1}\right] .
$$

Substituting in Eq. (4), we get

$$
\left\langle V_{z z}\right\rangle=V_{z z}+\frac{\hbar V_{z z z z}}{2}\left[\frac{1}{2}+\frac{1}{e^{\theta / T}-1}\right]\left[\frac{1}{\sqrt{k_{z}}}-\frac{1}{\sqrt{k_{x}}}\right],
$$

where $\theta=\hbar \omega / k_{B}$ is the Einstein temperature. It may be seen in the above equation that even when $V_{z z}$ is absent, $\left\langle V_{z z}\right\rangle$ has a finite value at $T=0$. This is a consequence of the zero-point 

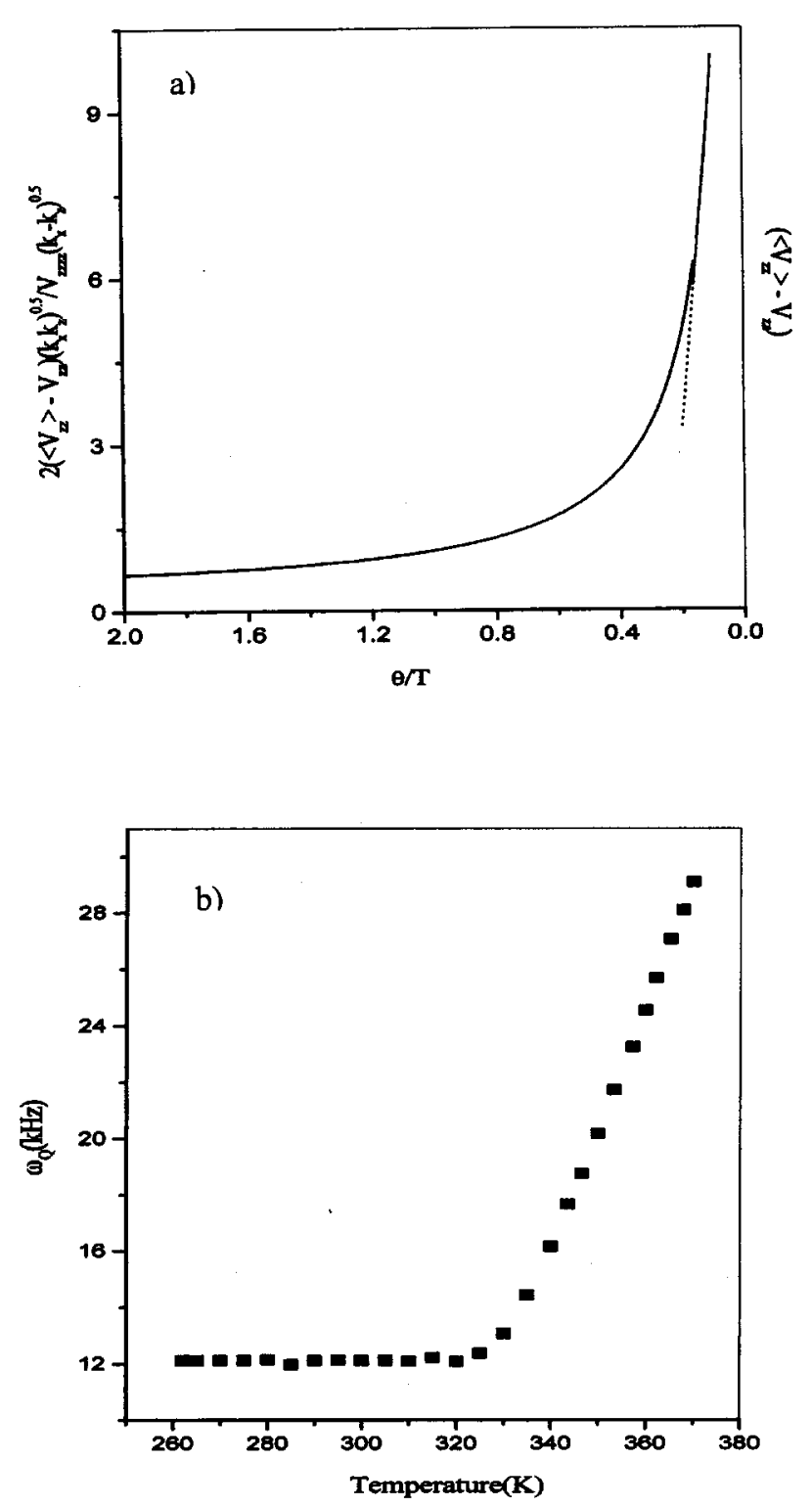

FIG. 3. (a) Variation of $2\left(\left\langle V_{z z}\right\rangle-V_{z z}\right)\left(\sqrt{k_{x} k_{z}}\right) / V_{z z z z}\left(\sqrt{k_{x}-k_{z}}\right)$ as a function of $\theta / T$ for a quantum oscillator. For comparison the temperature variation of $\left(\left\langle V_{z z}\right\rangle-V_{z z}\right)$ for a classical oscillator (dotted line) is also shown. (b) Temperature variation of the quadrupolar coupling constant $\omega_{Q}$ as determined from the fit to the spin-lattice relaxation data (solid line).

energy $\hbar \omega / 2$ for a quantum oscillator. This is the most notable difference in the expressions for $\left\langle V_{z z}\right\rangle$ for the quantum [Eq. (13)] and classical [Eq. (6)] oscillators.

Equation (13) can be rewritten as

$$
\frac{2\left(\left\langle V_{z z}\right\rangle-V_{z z}\right)}{V_{z z z z}} \frac{\sqrt{k_{x} k_{z}}}{\sqrt{k_{x}-k_{z}}}=\hbar\left[\frac{1}{2}+\frac{1}{e^{\theta / T}-1}\right]
$$

The variation of $2\left(\left\langle V_{z z}\right\rangle-V_{z z}\right)\left(\sqrt{k_{x} k_{z}}\right) / V_{z z z z}\left(\sqrt{k_{x}-k_{z}}\right)$ as a function of $\theta / T$ is shown as a solid line in Fig. 3(a). For comparison, the quantity $\left(\left\langle V_{z z}\right\rangle-V_{z z}\right)$ of the classical oscillator as a function of $\theta / T$ is also shown in Fig. 3(a) as a dotted line.

For $T \ll \theta$, Eq. (13) may be rewritten as

$$
\left\langle V_{z z}\right\rangle=V_{z z}+\frac{\hbar V_{z z z z}}{2}\left[\frac{1}{2}+e^{-\theta / T}\right]\left[\frac{1}{\sqrt{k_{z}}}-\frac{1}{\sqrt{k_{x}}}\right] .
$$

On the other hand, for $T \gg \theta$, Eq. (13) yields

$\left\langle V_{z z}\right\rangle=V_{z z}+\frac{\hbar V_{z z z z}}{4}\left[\frac{1}{\sqrt{k_{z}}}-\frac{1}{\sqrt{k_{x}}}\right]+\frac{\hbar V_{z z z z} T}{2 \theta}\left[\frac{1}{\sqrt{k_{z}}}-\frac{1}{\sqrt{k_{x}}}\right]$.

The temperature dependence [Eq. (16)] is equivalent to that of the classical oscillator [Eq. (6)] and shows a linear dependence.

The differences in the temperature dependence of the average field gradient for the quantum and classical oscillators is clearly brought out in Fig. 3(a). It is only at high temperatures $T>\theta$ that the linear dependence of $V_{z z}$ on temperature is recovered. At low temperatures the quantum anisotropic oscillator model predicts that the average field gradient is almost temperature independent with a limiting value of $V_{z z}+\left(\hbar V_{z z z z} / 4\right)\left[1 / \sqrt{k_{z}}-1 / \sqrt{k_{z}}\right]$ at absolute zero. Thus in situations where $V_{z z}$ is intrinsically small a quadrupolar splitting may not be observed at low temperatures, especially if the magnitude of the anisotropy of the force constants is small. At higher temperatures $(T>\theta)$, however, a quadrupolar splitting may be observed with the splitting showing a linear temperature dependence. This behavior is similar to that observed in the temperature variation of the ${ }^{7} \mathrm{Li} \mathrm{NMR}$ of $\mathrm{Cd}_{0.75} \mathrm{PS}_{3} \mathrm{Li}_{0.5}$ (PEO) as well as in the Li-crown ethers. ${ }^{19}$ The anisotropic quantum oscillator model is therefore able to explain this rather unusual observation of quadrupolar splitting at high temperature and its absence at low temperatures.

Spin-lattice relaxation of ${ }^{7} \mathrm{Li}$ nuclei are known to be dominated by quadrupolar relaxation. The spin-lattice relaxation rates are related to the real part of the spectral density function $^{20}$ via Eq. (17):

$$
1 / T_{1}=\omega_{Q}^{2}(T)\left[J\left(\omega_{0}\right)+4 J\left(2 \omega_{0}\right)\right],
$$

where $\omega_{Q}$ is the quadrupole frequency. An increase in the strength of the quadrupolar interaction, the magnitude of $\omega_{Q}$, would lead to a decrease in $T_{1}$. It is observed from the ${ }^{7} \mathrm{Li}$ NMR spectra (Fig. 1) that $\omega_{Q}$ increases linearly with temperature above $330 \mathrm{~K}$. The origin of the decrease in $T_{1}$, above $330 \mathrm{~K}$, can therefore be explained by this increase in magnitude of $\omega_{Q}$. For an Einstein oscillator $\omega_{Q}$ would be essentially temperature independent at low temperatures, but at high temperatures would exhibit a linear increase with temperature [Fig. 3(a)]. Such a model would be able to explain the unusual observation of the decrease in the spinlattice relaxation rates at high temperatures in the IPE without having to invoke the existence of another type of motion or degree of freedom at high temperatures through which relaxation can occur.

The linearity of the magnetization recovery in the "inversion-recovery" experiment indicates that the mechanism of magnetization recovery is characterized by a single correlation time at all temperatures. The relaxation data of $\mathrm{Cd}_{0.75} \mathrm{PS}_{3} \mathrm{Li}_{0.5}$ (PEO) in the temperature range $250-330 \mathrm{~K}$ could be analyzed using the Bloembergen-Purcell-Pound (BPP) model $^{23}$ in which the correlation function $g(t)$ decays 
as a single exponential, $g(t)=\exp (-t / \tau)$. The BPP expression for the spin-lattice relaxation times is given by

$$
\begin{aligned}
1 / T_{1} & =K(T)\left[J\left(\omega_{0}\right)+4 J\left(2 \omega_{0}\right)\right] \\
& =K(T)\left[\tau_{c} /\left(1+\omega_{0}^{2} \tau_{c}^{2}\right)+4 \tau_{c} /\left(1+4 \omega_{0}^{2} \tau_{c}^{2}\right)\right],
\end{aligned}
$$

where $K$ depends on the particular spin interaction responsible for the relaxation. In the Li-IPEs, $K(T)$ may be identified with $\omega_{Q}^{2}$ as in Eq. (17). In fitting the above expression [Eq. (18)] to the experimental relaxation data (Fig. 2) the temperature dependence of the correlation time $\tau_{c}$ was assumed to follow a VTF dependence $\tau_{c}=\tau_{o} \exp \left[-E_{a} / k(T\right.$ $\left.-T_{0}\right)$ ]. It was found, however, that the best fits were always obtained for $T_{0}=0$ in the VTF expression, indicating that $\tau_{c}$ has an Arrhenius temperature dependence. The parameters $K$, $\tau_{o}$, and $E_{a}$ were floated during the fitting exercise. The solid line in Fig. 2 is a fit to Eq. (18) obtained for $E_{a}$ $=6.55 \mathrm{kcal} / \mathrm{mol}$ and $\tau_{o}=7.3 \times 10^{-14} \mathrm{~s}$. The values of $\omega_{Q}$ which gave the best fits at each temperature are plotted in Fig. 3(b). [The dashed lines in Fig. 2 are the fitted $T_{1}$ values assuming that $K(T)$-i.e., $\omega_{Q}$-in Eq. (18) is independent of temperature.] The temperature variation of the quadrupolar coupling constant $\omega_{Q}$ as determined from the spin-lattice relaxation data bears a striking resemblance to that calculated using the anisotropic quantum oscillator model. Both show that $\omega_{Q}$ values are independent of temperature at low temperatures, but at higher temperatures show a linear increase with increasing temperature.

\section{CONCLUSION}

The appearance of satellite peaks due to quadrupolar interactions at high temperatures in the ${ }^{7} \mathrm{Li}(I=3 / 2)$ NMR spectra in an intercalated polymer electrolyte $\mathrm{Cd}_{0.75} \mathrm{PS}_{3} \mathrm{Li}_{0.5}(\mathrm{PEO})$ is discussed. This temperature range corresponds to the region where the conductivity data show appreciable mobility of the confined Li ions. This unusual behavior is also reflected in the temperature variation of the spin-lattice relaxation rates. The spin-lattice relaxation shows a minima at $300 \mathrm{~K}$, but above $330 \mathrm{~K}$, the temperature at which the quadrupolar satellites appear in the ${ }^{7} \mathrm{Li}$ spectra, starts decreasing.

The observed increase in the ${ }^{7} \mathrm{Li}$ quadrupolar coupling constant $\omega_{Q}$ with temperature may be explained as arising from a change in the time average of the electric field gradient seen by a ${ }^{7} \mathrm{Li}$ nuclei vibrating anisotropically about its equilibrium value. The anisotropy in the force constants would arise naturally if the $\mathrm{Li}$ ions are coordinated to the ether oxygens of the intercalated PEO in a crown-ether-like geometry as discussed in the preceding paper. ${ }^{16}$ We have shown here that an anisotropic quantum Einstein oscillator model for quadrupolar coupling can reproduce the observed temperature dependence of $\omega_{Q}$. The model predicts that at low temperatures the quadrupolar coupling would be essentially temperature independent, but at higher temperatures would increase linearly with temperature. The anisotropic quantum oscillator model is therefore able to explain the observed quadrupolar splitting at high temperature and its absence at low temperatures in the ${ }^{7} \mathrm{Li}$ NMR spectra of the intercalated polymer electrolyte.

${ }^{1}$ P. V. Wright, Br. Polym. J. 7, 319 (1975).

${ }^{2}$ Solid State Electrochemistry, edited by P. G. Bruce (Cambridge University Press, Cambridge, England, 1995).

${ }^{3}$ F. Croce, G. B. Appetecchi, L. Persi, and B. Scrosati, Nature (London) 394, 456 (1998).

${ }^{4}$ F. M. Gray, Solid Polymer Electrolytes: Fundamentals and Technological Applications (VCH, New York, 1991).

${ }^{5}$ Polymer Electrolyte Reviews, edited by J. R. MacCallum and C. A. Vincent (Elsevier, New York, 1987 and 1989), Vol. 1 and 2.

${ }^{6}$ Solid State Electrochemistry, edited by P. G. Bruce (Cambridge University Press, Cambridge, England, 1995).

${ }^{7}$ S. G. Greenbaum, Polym. Adv. Technol. 4, 172 (1992).

${ }^{8}$ J. P. Donoso, T. J. Bonagamba, H. C. Panepucci, and L. N. Oliveira, J. Chem. Phys. 98, 10026 (1993).

${ }^{9}$ M. Forsyth, M. Garcia, D. R. Macfarlane, S. Ng, M. E. Smith, and J. H. Strange, Solid State Ionics 86-88, 1365 (1996).

${ }^{10}$ S. H. Chung, K. R. Jeffrey, and J. R. Stevens, J. Chem. Phys. 94, 1803 (1991).

${ }^{11}$ S. H. Chung, K. R. Jeffrey, and J. R. Stevens, Phys. Rev. B 51, 2826 (1995).

${ }^{12}$ S. H. Chung, K. R. Jeffrey, and J. R. Stevens, J. Chem. Phys. 108, 3360 (1998).

${ }^{13}$ S. Wong, S. Vasudevan, R. A. Vaia, E. P. Giannelis, and D. B. Zax, J. Am. Chem. Soc. 117, 7568 (1995)

${ }^{14}$ S. Wong, R. A. Vaia, E. P. Giannelis, and D. B. Zax, Solid State Ionics 86-88, 547 (1996).

${ }^{15}$ D. K. Yang and D. B. Zax, J. Chem. Phys. 110, 5325 (1999).

${ }^{16}$ N. Arun, S. Vasudevan, and K. V. Ramanathan, J. Chem. Phys. 119, 2840 (2003), preceding paper.

${ }^{17}$ P. Jeevanandam and S. Vasudevan, Chem. Mater. 10, 1276 (1998)

${ }^{18}$ P. Jeevanandam and S. Vasudevan, J. Chem. Phys. 109, 8109 (1998).

${ }^{19}$ T. Pietrass and P. K. Burkert, Magn. Reson. Chem. 31, 709 (1993).

${ }^{20}$ A. Abragam, Principles of Nuclear Magnetism (Oxford University Press, London, 1961).

${ }^{21}$ T. K. Halstead, J. Chem. Phys. 53, 3427 (1970).

${ }^{22}$ C. C. Tannoudji, B. Diu, and F. Laloe, Quantum Mechanics (Wiley, Paris, 1977), Vol. 1.

${ }^{23}$ N. Bloembergen, E. M. Purcell, and R. Pound, Phys. Rev. 73, 679 (1948). 